\title{
Spiritual Activation in Very Elderly Individuals Assessed as Heart Rate Variability and Plasma IL/10/IL-6 Ratios
}

\author{
Akira Kurita, ${ }^{1}$ MD, Bonpei TaKase, ${ }^{2} \mathrm{MD}$, Naosuke Shinagawa, ${ }^{3} \mathrm{MD}$, Eitaro Kodani, ${ }^{4} \mathrm{MD}$, \\ Kaoru OKada, ${ }^{4}$ MD, Shinichiro Iwahara, ${ }^{4}$ MD, Yoshiki Kusama, ${ }^{4}$ MD, and Hirotsugu Atarashi, ${ }^{4}$ MD
}

\begin{abstract}
SUMMARY
Proinflammatory cytokine responses might occur in elderly individuals with cardiovascular (CV) disease, cerebrovascular (CVA) disease, and/or pulmonary disease (PD). Spiritual activation is an important coping mechanism, since psychiatric depression is an important risk factor for these individuals.

Thirty-three very elderly individuals ( $87 \pm 8$ years) with previous CVD, CVA and/or PD participated in weekly 30 minute sermons by chaplains for over 20 months of chaplain liturgy (CL group). All underwent Holter ECG during the procedures and cardiac autonomic activities were assessed by maximum entropy analysis. Plasma IL-10 and IL-6 levels were compared with 26 age-matched ( $85 \pm 10$ years) individuals who did not participate in these activities (non-CL group). Both high frequency (HF) and pNN50 of heart rate variability (HRV) were higher in the CL group than in the non-CL group (HF, $190 \pm 55$ versus $92 \pm 43 \mathrm{nu}, P<0.05$; pNN50, $10.5 \pm 16 \%$ versus $3.6 \pm 3.8 \%, P<0.05$ ), whereas $\mathrm{LF} / \mathrm{HF}$ was lower $(1.4 \pm 1.5$ versus $2.2 \pm 2.8, P<0.05)$. Levels of IL-10/IL-6 were higher in the CL group $(3.96 \pm 5.0$ versus $1.79 \pm 1.6, P<0.05)$. Hospitalization rates due to CVD and/or PD were lower in the CL group than in the nonCL group (4/33 versus $11 / 26, P<0.05)$.

We conclude that spiritual activation can modify proinflammatory cytokines and suppress CVD, CVA and/or PD via vagal modifications. Spiritual activation might be helpful for health in these very elderly individuals. (Int Heart $\mathbf{J}$
\end{abstract} 2011; 52: 299-303)

Key words: Spiritual activation, Heart rate variability, IL-10/IL-6

$\mathrm{E}$ motional distress is a frequent feature of patients in geriatric nursing homes. Several observational studies have found that depression and emotional distress are associated with cardiovascular diseases (CVD), cerebrovascular disease (CVA) and pulmonary disease (PD), especially aspiration pneumonia, ${ }^{1-6)}$ because the number of these elderly patients is increasing. By the year 2025, individuals over 65 years of age will comprise about $25 \%$ of the entire Japanese population and solitary death rates among the aging are increasing. ${ }^{7)}$ Therefore, activation of the emotions via spirituality is an important health care strategy for preventing dementia in geriatric nursing homes. Chaplains, doctors and/or local elder community choral groups can provide spiritual activation activities. Although chaplain liturgies (CL) have been delivered at our geriatric welfare home for over 25 years, whether or not this really benefits spiritual states, autonomic nerve activities and/ or the physical health of the very elderly remains unknown. The present study evaluates the effects of CL on autonomic nerve activities by measuring heart rate variability (HRV), which is a principal method of assessing cardiac autonomic tone. Low HRV that reflects high sympathetic and/or low parasympathetic tone is a powerful independent predictor of mortality among those with not only CVD but also with chronic morbidities. ${ }^{8-11)}$ We also measured ratios of IL- 6 and IL-10, be- cause IL-6 is a key signaling pathway that is implicated in aging and chronic morbidity ${ }^{12-15)}$ and IL-10 has multifaceted antiinflammatory properties that lead to suppressed cytokine production as well as the inhibition of apoptosis after infection, ${ }^{16-18)}$ and aspiration pneumonia together with heart failure are the main causes of death in geriatric nursing homes.

\section{MeTHODS}

Study sample: Fifty-nine individuals $(87.3 \pm 9.6$ years $)$ were referred by a doctor to our geriatric welfare home due to having confirmed subclinical CVD, CVA, and/or PD. We assigned them to either a group that attended CL ( $n=33$, age; $87 \pm 5$ years) or a group that did not (non-CL; $n=26$, age; $85 \pm 10$ years). Those in the CL group attended CL at least once each week for over 20 months, and the other group had a similar physiological background to the CL group but did not attend CL. To avoid possible confounding effects, we excluded those with concomitant conditions with known independent effects on autonomic activities such as clinical evidence of autonomic neuropathy, any acute inflammatory disease, acute coronary ischemia, or other acute illnesses or malignant diseases that required hospitalization. The study proceeded as part of routine

From ${ }^{1}$ Special Nursing Home for Elderly, Fukuinkai Clinic, Tokyo, ${ }^{2}$ Department of Emergency Medicine, National Defense Medical College, Saitama, ${ }^{3}$ Shinagawa Clinic, and ${ }^{4}$ Department of Internal Medicine, Division of Cardiology, Nippon Medical School, Tama Nagayama Hospital, Tokyo, Japan.

This study was performed with the support of the Mitsukoshi Kousei Jigyoudan.

Address for correspondence: Akira Kurita, MD, Fukuinkai Clinic, 1932 Notsuda, Machida City, Tokyo 195-0063, Japan.

Received for publication March 28, 2011.

Revised and accepted June 2, 2011. 
care in accordance with the principles of the Declaration of Helsinki. ${ }^{19)}$ All participants underwent a standard clinical evaluation that included a medical history, neurological examination, and cognitive function assessment. Dementia was diagnosed by consensus at a conference of physicians, nurses, and care workers at the time of admission based on the results of the Mini-Mental State Examination (score range from 1 to 5). ${ }^{20)}$ We compared physical parameters such as age, gender, level of dementia, CT findings, and complication with previous illnesses, namely, CVA, diabetes mellitus (DM), congestive heart failure and types of medications, between the CL and non-CL groups. We also compared rates of hospitalization events due to aspiration pneumonia and/or heart failure between the two groups.

Chaplain liturgies $(\mathbf{C L})$ : Five experienced and licensed chaplains delivered liturgies once each week for about 30 minutes from 15.30 to 16.00 for over 10 years. During CL, hymns were sung with organ accompaniment before and after a sermon (Figure 1). The issues addressed in each sermon were selected from the bible and the chaplains delivered a simple but important message to the participants. Attempts were made to include at least one popular hymn so that participants could sing along with the organ accompaniment.

Heart rate variability (HRV): We obtained HRV using an ambulatory Holter ECG system with a two-channel (Fukuda Denshi FMD-150) ECG recorder. Spectral power results were obtained from the first 5-minute segments with a total spectrum of 320 points. Over a bandwidth ranging from 0.01 to $1.0 \mathrm{~Hz}$, the low-frequency (LF) band $(0.04$ to $0.15 \mathrm{~Hz})$, the high-frequency (HF) band $(0.15$ to $0.40 \mathrm{HZ})$, and all power values were calculated using GMS Chiram software. ${ }^{21)}$ The LF and $\mathrm{HF}$ components were measured in $\mathrm{ms}^{2}$ and are expressed as power. The HF component mainly reflects parasympathetic activity, whereas LF/HF mainly reflects sympathetic activity and sympatho-vagal balance. ${ }^{8}$ Time domain was analyzed using the pNN50 (ratio (\%) of successive RR-interval differences > $50 \mathrm{~ms}$ ), rMSSD (the square root of the mean of the sum of the squares of differences between adjacent intervals), and SDNN (standard deviation of all $\mathrm{NN}$ intervals in all 5-minute segments of entire recording). ${ }^{8)}$ These parameters were calculated using the same software as the frequency domain analysis and

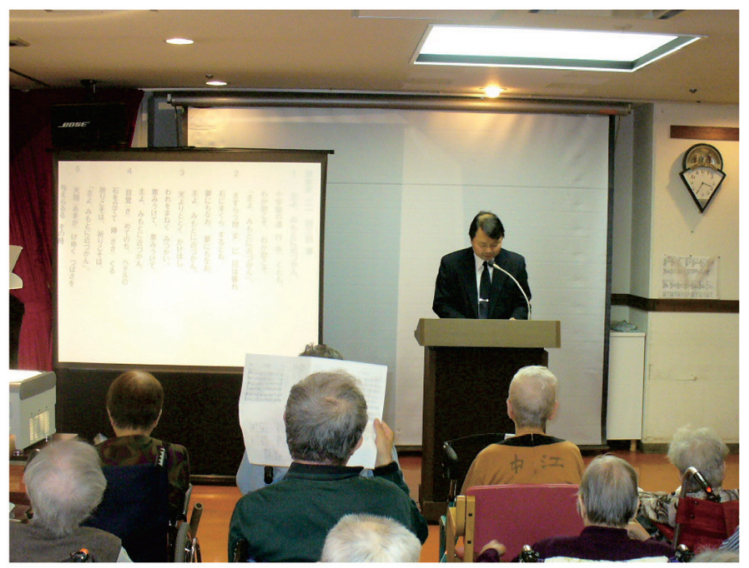

Figure 1. Chaplain delivering sermon. reflect parasympathetic activity from short electrocardiographic recordings since recent advances in software development allow predictions of mortality from all causes among elderly patients. ${ }^{22,23)}$ Heart rate variability was measured before (14:30 to $15: 30)$, during (15:30 to $16: 10)$, and after (16:10 to $17: 10$ ) CL. The HRV of the CL group was the average of all values in these 3 conditions. Autonomic nerve activities were measured in the non-CL group over the same time period as described for the CL group.

Blood samples: Blood samples were collected in the morning in the fasting state within one week before obtaining Holter ECG recordings and plasma from both groups was stored at $-70^{\circ} \mathrm{C}$ in a single batch until analysis. Plasma cytokines (both IL-6 and IL-10) were measured using the HPLA method and the intra-assay coefficient of variance was $<5 \%$ for all assays. Statistical analysis: All parameters were analyzed by repeated measures of ANOVA and are presented as the mean \pm SD. The $\mathrm{CL}$ and non-CL groups were compared using an unpaired $t$ test and two-way analysis of variance with repeated measurements. A $P$ value of $<0.05$ was considered to indicate statistical significance.

\section{RESULTS}

The clinical characteristics did not significantly differ between the two groups (Table I). The average dementia grades were $3.2 \pm 0.5$ and $3.2 \pm 0.7$ in the CL and non-CL groups, respectively. Prescribed medications and the incidence of CVA, $\mathrm{DM}$, and congestive heart failure also did not differ significantly between the groups.

Among frequency domain HRV, averaged HF was significantly higher ( $190 \pm 55$ versus $92 \pm 43 \mathrm{~ms}, P<0.05)$, whereas averaged $\mathrm{LF} / \mathrm{HF}$ that is thought to reflect sympathetic tone was significantly lower $(1.4 \pm 1.5$ versus $2.2 \pm 2.8, P<0.05)$ in the $\mathrm{CL}$ group than in the non-CL group (Table II).

Among time domain HRV parameters, PNN50 was significantly higher $(10.5 \pm 16 \%$ versus $3.6 \pm 3.8 \%, P<0.05$; Table II) and rMSSD was slightly higher (32.4 \pm 26 versus 25.4

Table I. Baseline Clinical Characteristics of Patients

\begin{tabular}{lcc}
\hline & CL & Non-CL \\
\hline Number of patients & 33 & 26 \\
Age & $87 \pm 5$ & $85 \pm 10$ \\
Male/Female & $6 / 27$ & $4 / 22$ \\
Hypertension & 4 & 3 \\
OMI & 3 & 5 \\
DM & 2 & 3 \\
Previous CVA & 11 & 10 \\
Previous PD & 3 & 2 \\
ACE & 7 & 6 \\
Ca-channel blocker & 3 & 6 \\
Nitrates & 3 & 3 \\
Diuretics & 3 & 3 \\
Sedatives & 2 & 1 \\
Grade of dementia & $3.5 \pm 2.5$ & $3.7 \pm 2.0$ \\
\hline
\end{tabular}

mean $\pm \mathrm{SD} . P$ was not significant for any factors. CL indicates chaplain liturgies; OMI, old myocardial infarction; DM, diabetes mellitus; CVA, cerebrovascular accident; PD, pulmonary disease; and ACE, angiotensinconverting enzyme inhibitor. 
Table II. Summary of Heart Rate Variability Indexes and Plasma IL10/ IL-6 Levels in the CL and Non-CL Groups

\begin{tabular}{lccccc}
\hline & CL & $n$ & Non-CL & $n$ & $P$ \\
\hline SDNN (ms) & $67 \pm 70$ & 33 & $60 \pm 73$ & 26 & NS \\
rMSSD (ms) & $32.4 \pm 26.0$ & 33 & $25.4 \pm 22$ & 26 & NS \\
pNN50 (\%) & $10.5 \pm 16.0$ & 33 & $3.6 \pm 3.8$ & 26 & $<0.05$ \\
HF (nu) & $190 \pm 55$ & 33 & $92 \pm 43$ & 26 & $<0.05$ \\
LF/HF & $1.4 \pm 1.5$ & 33 & $2.2 \pm 2.8$ & 26 & $<0.05$ \\
IL-6 (pg/mL) & $8.9 \pm 1.8$ & 25 & $9.5 \pm 2.5$ & 20 & NS \\
IL-10 (pg/mL) & $34.2 \pm 18.5$ & 25 & $16.8 \pm 4.3$ & 20 & $<0.10$ \\
IL-10/IL-6 & $3.96 \pm 5.0$ & 25 & $1.79 \pm 1.6$ & 20 & $<0.05$ \\
\hline
\end{tabular}

SDNN indicates standard deviation of normal RR intervals; rMSSD, square root of the mean of the squared differences between adjacent normal RR intervals; pNN50, percentage of differences between adjacent normal RR intervals > 50 milliseconds; LF, low frequency; HF, high frequency; and IL, interleukin.

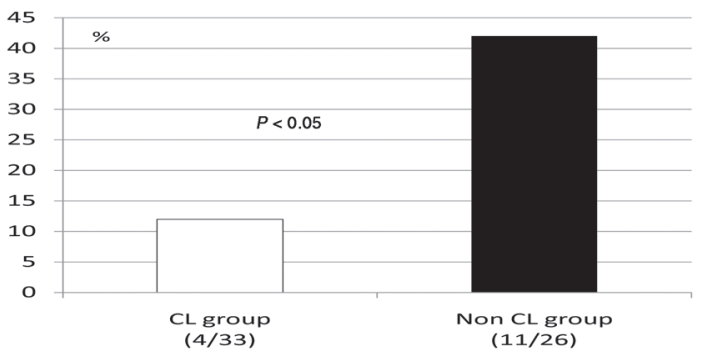

Figure 2. Comparison of hospitalization incidence between CL and nonCL groups.

$\pm 22 \mathrm{~ms}, P=0.14)$ in the CL group than in the non-CL group, whereas SDNN did not differ significantly (Table II).

Plasma ratios of inflammatory and anti-inflammatory cytokines are shown in the bottom line in Table II. Although IL-6 was not significantly different between the two groups, IL-10 was higher in the CL group than in the non-CL group (34.2 \pm 18.5 versus $16.8 \pm 4.3 \mathrm{pg} / \mathrm{mL}, P<0.10)$. The IL-10/IL-6 was significantly higher in the CL group than in the non-CL group $(3.96 \pm 5.0$ versus $1.79 \pm 1.6, P<0.05)$. The incidence of hospitalization mostly due to aspiration pneumonia and/or heart failure is shown in Figure 2. The event rates of aspiration pneumonia and/or heart failure were lower in the CL group than in the non-CL group (4/33 versus $11 / 26, P<0.05)$.

\section{Discussion}

The present findings suggest that CL could positively activate parasympathetic tone by increasing plasma levels of antiinflammatory rather than inflammatory cytokines. Such mobilization of autonomic tone by CL might attenuate the psychological stimulation of autonomic tone associated with chronic morbidity even among ill octogenarians with dementia, since events rates of aspiration pneumonia and/or heart failure were lower in the CL group than in the non-CL group.

Ambulatory electrocardiographic monitoring is an inexpensive and noninvasive method of assessing HRV that is becoming popular for estimating autonomic function in clinical cardiology. ${ }^{8-10,24)}$ It is generally considered the most appropriate standard method of assessing quantitative markers of cardiac autonomic activity, according to the Task Force of the European Society of Cardiology (ESC) and the North American Society of Pacing Electrophysiology (NASPE). ${ }^{8)}$ Recent advances in Holter ECG systems have produced small, light equipment such as the Fukuda-Denshi FD 50, which weighs only $150 \mathrm{~g}$. This system was therefore appropriate for measuring HRV in elderly patients with dementia without causing distress. According to the Task Force of the ESC and NASPE, short-term 2- to 5-minute recordings can reflect autonomic nervous tone by either spectrum calculations of frequency domain or time domain methods. ${ }^{8)}$ We used the maximum entropy method calculation (Mem Calc) incorporated within Chiram software ${ }^{21)}$ to evaluate autonomic function, because this system could analyze both spectral and time domains of HRV by recording 5-minute ECG segments using the exponential method of power spectral density analysis. ${ }^{8,23)}$ The present study found that pNN50 (time domain parameters) and HF spectra (main frequency domain parameter) were significantly higher in the CL group than the non-CL group, whereas other HRV parameters obtained by both time and frequency domain parameters did not significantly differ. The higher values of pNN50\% and $\mathrm{HF}$, and the significantly lower values of LF/HF, suggested that spiritual activation induced by attending sermons and singing hymns activated parasympathetic tone in the CL group to some extent.

That the immune system plays a critical role at every stage of atherosclerotic plaque has become appreciated, since atherosclerosis is considered an essentially inflammatory phenomenon. ${ }^{25-27)}$ Extrapolating from middle to old age is problematic for various reasons. ${ }^{28)}$ The usual risk factors for cardiovascular events change because total serum cholesterol and LDL-cholesterol are weaker predictors of future events among the elderly. ${ }^{29,30)}$ Indeed, low cholesterol levels in some elderly populations are associated with elevated risk. ${ }^{31,32)}$ Because of these recent considerations, we measured the spiritual effects of CL and hymns on serum cytokine IL-6 and IL-10 levels. Cesari, et al reported that IL- 6 is a better predictor of incident coronary disease than $\mathrm{CRP}^{32}{ }^{32}$ because its relationship with incident stroke is closer for higher relative risks. In contrast, serum levels of the potent anti-inflammatory cytokine IL-10 are decreased in patients with atherosclerotic disease. Activated monocytes/macrophages and lymphocytes secrete IL-10, ${ }^{33)}$ which has multifaceted anti-inflammatory properties, including the inhibition of the prototypic proinflammatory transcription nuclear factor that leads to suppressed cytokine production, ${ }^{34)}$ reduced tissue factor expression ${ }^{35)}$ and the inhibition of macrophage and monocyte apoptosis after infection. ${ }^{36)}$ We therefore measured the IL10/IL-6 ratio that responds to inflammatory insult and exaggerates these responses. The IL-10/IL-6 was significantly higher in the $\mathrm{Cl}$ group than in the non-CL group. These results suggest that spiritual activation by participating in CL sermons and singing hymns stabilizes proinflammatory pathways and activates the immune system. This would help to prevent respiratory infections and or vascular inflammation during the healing process and stabilize vascular thrombus. These effects through cytokines can activate beneficial anti-inflammatory effects on the central nervous system as well as hypothalamic-pituitary pathways. ${ }^{37)}$ The central nervous system receives sensory input from the immune system possibly through both humoral and neural routes. The immune system 
might detect microbial invasion and produce factors that relay such information to the brain. ${ }^{38)}$ Increased efferent signals in the vagus nerve suppress peripheral cytokine release through macrophage nicotinic receptors and cholinergic anti-inflammatory pathways that might have resulted in fewer individuals in the CL group being hospitalized.

However, the present study has several limitations. First, the sample size might be insufficient. The second limitation is the single assessment of cytokine levels and HRV measurements, with blood samples being collected about 1 week and 3 days before HRV testing to determine cytokine production due to insufficient manpower. Indeed to arrive at a precise conclusion, plasma cytokines should be measured during fasting for the Holter ECG procedure. Although evidence suggests that the magnitude of both stimulated cytokine production by peripheral leukocytes and vagal control of heart rate is subject to individual variations and that such variability is stable across time, multiple simultaneous assessments over time would provide a more reliable measure of individual differences. Hopefully, if other activities that can induce spiritual activation, such as music therapy, ${ }^{39,40)}$ can be compared, then more useful information will be generated, since the song associated with the popular children's animated show "AnPan-Man" was found to provide spiritual activation to many people affected by the recent magnitude 9.0 earthquake and tsunami in northern Japan, because many people called radio stations and said they were "Very encouraged" and "Children started laughing for the first time since the disaster struck". ${ }^{41)}$ Our data suggest that spiritual activation can modify proinflammatory cytokines and suppress heart failure and/or respiratory infection via vagal nerve modifications.

We believe that spiritual activation might thus be helpful for improving the quality of life in these very elderly individuals.

\section{ACKNOWLEDGMENTS}

We would like to especially thank Rev. Eiji Seto, Rev. Zenichirou Hiramoto, Rev. Yoshiharu Akinaga, Rev. Shigeru Kobayashi and Rev. Osamu Sadaie for their kind chaplain liturgies.

\section{REFERENCES}

1. Carney RM, Rich MW, Tevelde A, Saini J, Clarke K, Jaffe AS Major depressive disorders in coronary artery disease. Am J Cardiol 1987; 60: 1273-5.

2. Glassman AH, Shapiro PA. Depression and the course of coronary artery disease. Am J Psychiatry 1998; 155: 4-11. (Review)

3. Mallik S, Krumholz HM, Lin ZQ, et al. Patients with depressive symptoms have lower health status benefits after coronary artery bypass surgery. Circulation 2005; 111: 271-7.

4. Lichtman JH, Bigger JT Jr, Blumenthal JA, et al. Depression and coronary heart disease: recommendations for screening, referral, and treatment: a science advisory from the American Heart Association Prevention Committee of the Council on Cardiovascular Nursing, Council on Clinical Cardiology, Council on Epidemiology and Prevention, and Interdisciplinary Council on Quality of Care and Outcomes Research: endorsed by the American Psychiatric Association. Circulation 2008; 118: 1768-75.

5. Arnold SV, Spertus JA, Ciechanowski PS, et al. Pscychosocial modulators of angina response to myocardial ischemia. Circula- tion 2009; 120: 126-33.

6. Gottlieb SS, Kop WJ, Ellis SJ, et al. Relation of depression to severity of illness in heart failure (from Heart Failure And a Controlled Trial Investigation Outcomes of Exercise Training [HF-ACTION]) Am J Cardiol 2009; 103: 1285-9.

7. Kokumin Eisei no Doukou. Vol 52 In Jinkou Doutai. Tokyo* Health and Welfare Statistics Association 2006; 33-7. (Japanese).

8. Heart rate variability: standards of measurement, physiological interpretation, and clinical use. Task Force of the European Society of Cardiology and the North American Society of Pacing and Electrophysiology. Circulation 1996; 93: 1043-65.

9. Anderson KP, Bigger JT Jr, Freedman RA. Electrocardiographic predictors in the ESVEM trial: unsustained ventricular tachycardia, heart period variability, and the signal-averaged electrocardiogram. Prog Cardiovasc Dis 1996; 38: 463-88. (Review)

10. Malik M. Heart rate variability. Curr Opin Cardiol 1998; 13: 3644. (Review)

11. Guzzetti S, Borroni E, Garbelli PE, et al. Symbolic dynamics of heart rate variability: a probe to investigate cardiac autonomic modulation. Circulation 2005; 112: 465-70.

12. Ershler WB. Interleukin-6: a cytokine for gerontologists. J Am Geriatr Soc 1993; 41: 176-81. (Review)

13. Opal SM, DePalo VA. Anti-inflammatory cytokines. Chest 2000; 117: 1162-72. (Review)

14. Kritchevsky SB, Cesari M. Pahor M. Inflammatory markers and cardiovascular health in older adults. Cardiovasc Res 2005; 66: 265-75. (Review)

15. Maggio M, Guralnik JM, Longo DL, Ferrucci L. Interleukin-6 in aging and chronic disease: a magnificent pathway. J Gerontol A Biol Scu Med Sci 2006; 61: 575-84. (Review)

16. Mallat Z, Besnard S, Duriez M, et al. Protective role of interleukin-10 in atherosclerosis. Circ Res 1999; 85: e17-24.

17. Tracey KJ. The inflammatory reflex. Nature 2002; 420: 853-9. (Review)

18. Murray DR, Freeman GL. Proinflammatory cytokines: predictors of a failing heart? Circulation 2003; 107: 1460-2.

19. Declaration of Helsinki (1964). BMJ 1996; 313: 1448-9.

20. Crum RM, Anthony JC, Bassett SS, Folstein MF. Populationbased norms for the Mini-Mental State Examination by age and education level. JAMA 1993; 269: 2386-91.

21. Ohtomo N, Terachi S, Tanaka Y, Tokiwano K, Kaneko N. New method of time series analysis and its application to Wolf's sunspot number data. Jpn J Appl Phys 1994; 33: 2821-31.

22. Dekker JM, Schouten EG, Klootwijk P, Pool J, Swenne CA, Kromhout D. Heart rate variability from short electrocardiographic recordings predicts mortality from all causes in middle-aged and elderly men. The Zutphen Study. Am J Epidemiol 1997; 145: 899-908

23. Pomeranz B, Macaulay RJ, Caudill MA, et al. Assessment of autonomic function in humans by heart rate spectral analysis. Am J Physiol 1985; 248: H151-3.

24. Huikuri HV, Perkiömäki JS, Maestri R, Pinna GD. Clinical impact of evaluation of cardiovascular control by novel methods of heart rate dynamics. Philos Transact A Math Phys Eng Sci 2009; 367: 1223-38. (Review)

25. Ross R. Atherosclerosis--an inflammatory disease. N Engl J Med 1999; 340: 115-26. (Review)

26. Libby P. Changing concepts of atherogenesis. J Intern Med 2000; 247: 349-58. (Review)

27. Libby P, Ridker PM, Maseri A. Inflammation and atherosclerosis. Circulation 2002; 105: 1135-43. (Review)

28. Manolio TA, Pearson TA, Wenger NK, Barrett-Connor E, Payne GH, Harlan WR. Cholesterol and heart disease in older persons and women. Review of an NHLBI workshop. Ann Epidemiol 1992; 2: 161-76.

29. Kronmal RA, Cain KC, Ye Z, Omenn GS. Total serum cholesterol levels and mortality risk as a function of age. A report based on the Framingham data. Arch Intern Med 1993; 153: 1065-73.

30. Larson MG. Assessment of cardiovascular risk factors in the elderly: the Framingham Heart Study. Stat Med 1995; 14: 1745-56. 
31. Curb JD, Abbott RD, Rodriguez BL, et al. Prospective association between low and high total and low-density lipoprotein cholesterol and coronary heart disease in elderly men. J Am Geriatr Soc 2004; 52: $1975-80$

32. Cesari M, Penninx BW, Newman AB, et al. Inflammatory markers and onset of cardiovascular events: results from the Health $\mathrm{ABC}$ study. Circulation 2003; 108: 2317-22.

33. Lacraz S, Nicod LP, Chicheportiche R, Welgus HG, Dayer JM. IL-10 inhibits metalloproteinase and stimulates TIMP-1 production in human mononuclear phagocytes. J Clin Invest 1995; 96 : 2304-10.

34. Arai T, Hiromatsu K, Nishimura H, et al. Endogenous interleukin 10 prevents apoptosis in macrophages during Salmonella infection. Biochem Biophys Res Commun 1995; 213: 600-7.

35. Pinderski LJ, Fischbein MP, Subbanagounder G, et al. Overexpression of interleukin-10 by activated $\mathrm{T}$ lymphocytes inhibits atherosclerosis in LDL receptor-deficient Mice by altering lymphocyte and macrophage phenotypes. Circ Res 2002; 90: 1064
71.

36. Tracey KJ. The inflammatory reflex. Nature 2002; 402: 853-9. (Review)

37. Blalock FE. Shared ligands and receptors as a molecular mechanism for communication between the immune and neuroendocrine systems. Ann N Y Acad Sci 1994; 741: 292-8. (Review)

38. Hainsworth R. Reflexes from the heart. Physiol Rev 1991; 71: 617-58. (Review)

39. Kurita A, Takase B, Okada K, et al. Music therapy in very elderly cerebral vascular disorders patients with dementia. J Natl Def Med Coll 2007; 32: 143-52. (Review)

40. Okada K, Kurita A, Takase B, et al. Effects of music therapy on autonomic nervous system activity, incidence of heart failure events, and plasma cytokine and catecholamine levels in elderly patients with cerebrovascular disease and dementia. Int Heart J 2009; 50: 95-110.

41. AnPan-man's march getting up hit chart in NHK radio. Available at: http://tail-tale.blogspot.com/2011_03_01_archive.html. 